\section{AXONAL EXCITABILITY PROPERTIES IN DRAVET'S SYNDROME REFLECT EFFECT OF LOSS OF SODIUM CHANNELS}

${ }^{2,1}$ Michelle A Farrar*, ${ }^{4,3}$ Susan E Tomlinson* ${ }^{3}$ James Howells, ${ }^{3}$ Cindy Shin-YI Lin, ${ }^{2}$ Kate Carey, ${ }^{3}$ Susanna B Park, ${ }^{5}$ Georgina Hollingsworth, ${ }^{2,1}$ John A Lawson, ${ }^{3,6}$ Matthew C Kiernan, ${ }^{5}$ Sam F Berkovic, ${ }^{3,6}$ David Burke, Ingrid E Scheffer. 'Sydney Children's Hospital, Randwick, NSW, Australia; ${ }^{2}$ University of NSW, Sydney, NSW, Australia; ${ }^{3}$ Department of Medicine, Central Clinical School, University of Sydney, Sydney, NSW, Australia; ${ }^{4}$ Department of Neurology, St Vincent's Hospital, Sydney, NSW, Australia; ${ }^{5}$ University of Melbourne, Melbourne, VIC, Australia; ${ }^{6}$ Department of Neurology, Royal Prince Alfred Hospital, Camperdown, NSW, Australia; "Joint first authors

\subsection{6/jnnp-2019-anzan.9}

Introduction Mutations in $S C N 1 A$ encoding the $\mathrm{Na}_{\mathrm{v}} 1.1$ subunit of the neuronal sodium channel underlie the devastating epilepsy of Dravet's syndrome. ${ }^{1}$ The mechanism by which $\mathrm{Na}_{\mathrm{v}} 1.1$ dysfunction causes seizures is not clear. In vitro and in silico channel evaluation can support mutation pathogenicity but cannot define the in vivo impact of channel dysfunction. Axonal excitability studies can show the pattern of single-channel dysfunction in disorders where the channel is peripherally expressed. ${ }^{2}$ This study was undertaken to determine whether axonal excitability studies could detect changes in Dravet's patients related to the condition or due to medication effect.

Methods Patients with Dravet's syndrome were recruited from clinics in Sydney and Melbourne and axonal excitability studies were performed. Excitability results were analysed in 3 age groups and compared to age-matched normal controls.

Results Twenty six patients (ages 2-46) were studied. Findings were most pronounced in patients aged 20-46 $(n=7)$ with $6.9 \%$ greater increase in threshold during hyperpolarization $(p=0.1), 7.6 \%$ greater threshold decrease on depolarization $(\mathrm{p}=0.005)$ and, in the recovery cycle, $19.7 \%$ reduction in superexcitability $(\mathrm{p}=0.002)$ and $26 \%$ reduction in subexcitability $(p=0.03)$. Axonal excitability studies resembled previously published changes seen in patients with sodium channel blockade caused by acute tetrodotoxin poisoning. ${ }^{3}$

Conclusions Changes in excitability of axonal membrane in Dravet's syndrome are consistent with a decrease in sodium channel function. As the affected channel in Dravet's syndrome is not peripherally expressed, the effect seen is likely due to the heavy anticonvulsant regime required to control epilepsy, combined with a progressive loss of sodium channel function that occurs with age.

\section{REFERENCES}

1. Meisler MH, O'Brien JE, Sharkey LM. Sodium channel gene family: epilepsy mutations, gene interactions and modifier effects. J Physiol 2010;588:1841-1848.

2. Tomlinson SE, Howells J, Burke D. In vivo assessment of neurological channelopathies: Application of peripheral nerve excitability studies. Neuropharmacology. 2018 Apr;132:98-107.

3. Kiernan MC, Isbister GK, Lin CS, Burke D, Bostock H. Acute tetrodotoxin-induced neurotoxicity after ingestion of puffer fish. Ann Neurol 2005;57:339-48.

\section{0 \\ NEUROIMAGING AND CSF FINDINGS IN AUTOIMMUNE ENCEPHALITIS WITH NEURONAL CELL SURFACE ANTIBODIES}

\footnotetext{
2,1 James Broadley* ${ }^{*}{ }^{2,1}$ Robb Wesselingh, ${ }^{3,4}$ Paul Beech, 1,5,6 Udaya Seneviratne, ${ }^{7,8}$ Katherine Buzzard, ${ }^{1,7,8}$ Helmut Butzkueven, ${ }^{2,1,8}$ Terence $0^{\prime}$ Brien, ${ }^{2,1,8}$ Mastura Monif. ${ }^{1}$ Neuroscience, Monash University, Melbourne, VIC, Australia; ${ }^{2}$ Neurology, Alfred Health, Melbourne, VIC, Australia; ${ }^{3}$ Radiology, Alfred Health, Melbourne, VIC, Australia; ${ }^{4}$ Radiology، Monash Health, Melbourne, VIC, Australia; ${ }^{5}$ Neurology, Monash Health, Mel, VIC, Australia; ${ }^{6}$ Neurology, St Vincent's Health, Melbourne, VIC, Australia; ${ }^{7}$ Neurology, Eastern Health, Melbourne, VIC, Australia; ${ }^{8}$ Neurology, Melbourne Health, Melbourne, VIC, Australia
}

10.1136/jnnp-2019-anzan. 10
Introduction Neuroimaging and CSF analysis compose essential steps in evaluating patients with autoimmune encephalitis (AE). No study has compared the magnitude and prognostic implications of these findings between different subtypes. Herein we examine cases of AE with neuronal cell surface antibodies, and contrast the results of early investigations.

Methods We performed medical records search from 20082018 in 5 Victorian hospitals. Cases of AE were established in accordance with diagnostic criteria by Graus et al. ${ }^{1}$ Clinical and laboratory data was collected. All neuroimaging was evaluated independently by a neuroradiologist.

Results We identified 52 patients with AE with neuronal cell surface antibodies (21 NMDAR, 27 VGKC, 3 AMPAR, 1 GABAb).

We found that among patients with anti-LGI1 antibodies the presence of abnormal CSF correlated with increased rates of mortality $(50 \%$ vs $0 \%)$. This effect was largely mediated by CSF lymphocytosis, which was present in 2 patients who both died within 12 months of diagnosis.

We found that the development of hippocampal atrophy was more common amongst patients with abnormal MRI findings, but in particular those with changes affecting the medial temporal lobe. This effect was evident both in the cases with anti-VGKC antibodies $(60 \%$ vs $18 \%, 75 \%$ vs $17 \%)$ and the cohort as a whole (56\% vs $15 \%, 83 \%$ vs $13 \%)$.

Conclusions This is the first study of its kind in Australia, and identifies some unique correlation between early investigation findings, and the clinical and radiological outcome. A larger cohort is required to determine if these findings are statistically significant.

\section{REFERENCE}

1. Graus F, Titulaer MJ, Balu R, Benseler S, Bien CG, Cellucci T, Cortese I, Dale RC, Gelfand JM, Geschwind M, Glaser CA, Honnorat J, Hoftberger R, lizuka T, Irani SR, Lancaster E, Leypoldt F, Pruss H, Rae-Grant A, Reindl M, Rosenfeld MR, Rostasy $K$, Saiz A, Venkatesan A, Vincent A, Wandinger KP, Waters P, Dalmau J. A clinical approach to diagnosis of autoimmune encephalitis. Lancet Neurol 2016:15:391-404

\section{MELBOURNE MOBILE STROKE UNIT HALVES WORKFLOW FOR ACUTE STROKE REPERFUSION THERAPY}

1,2 Henry Zhao*, 1,2 Skye Coote, ${ }^{3}$ Francesca Langenberg, 1,2Damien Easton, ${ }^{4}$ Michael Stephenson, ${ }^{4}$ Karen Smith, ${ }^{4}$ Stephen Bernard, ${ }^{3,2}$ Patricia Desmond, ${ }^{3,2}$ Peter Mitchell, 1,2 Bernard Yan, 1,2 Bruce CV Campbell, 1,2 Mark Parsons, ${ }^{1,2}$ Geoffrey A Donnan, ${ }^{1,2}$ Stephen M Davis. ${ }^{1}$ The Melbourne Brain Centre and Department of Neurology, Royal Melbourne Hospital, Melbourne, VIC, Australia; ${ }^{2}$ Department of Medicine and Radiology, The University of Melbourne, Melbourne, VIC, Australia; ${ }^{3}$ Department of Radiology, Royal Melbourne Hospital, Melbourne, VIC, Australia; ${ }^{4}$ Ambulance Victoria, Melbourne, VIC, Australia

\subsection{6/jnnp-2019-anzan. 11}

Background The Melbourne Mobile Stroke Unit (MSU) utilises a specialised ambulance with on-board CT scanner and multidisciplinary team to provide on-scene imaging, treatment and triage for central Melbourne, Australia. We describe the operational impact of the MSU on commencement of acute reperfusion therapy.

Methods Data from the first 12 months of operation were collected for all patients receiving reperfusion therapy from November 2017. Workflow times were compared to contemporary published Australian data and historical controls from Royal Melbourne Hospital. 
Results In the first calendar 12 months of operation, the Melbourne MSU operated 30.5 service weeks and provided prehospital thrombolysis (tPA) to $\mathrm{n}=52$ patients (44\% of eligible infarcts) and directed $n=33$ patients for endovascular thrombectomy, of which $48 \%$ required bypass from the closest nonthrombectomy hospital. The overall median onset-to-tPA for MSU patients was 97.5 mins compared to the Australian metropolitan median of 150 mins. Thrombolysis in the first 'golden hour' increased to $13.5 \%$ from $3.3 \%$ in-hospital. Median onset-to-groin for MSU patients receiving EVT was 162 mins compared to 234 mins from historical controls.

Discussion Prehospital treatment and triage using the Mobile Stroke Unit in metropolitan Melbourne resulted in substantial improvements in commencement of reperfusion therapy. Workflow times are approximately halved for thrombolysis and endovascular thrombectomy respectively. Prehospital thrombolysis also allowed a $>400 \%$ increase in the proportion of treatment in the first 'golden hour'.

\section{MECHANICAL THROMBECTOMY IN PEDIATRIC STROKE: SYSTEMATIC REVIEW, INDIVIDUAL PATIENT-DATA META-ANALYSIS, AND CASE SERIES}

\begin{abstract}
${ }^{1,2}$ Kartik Bhatia, ${ }^{1}$ Hans Kortman, ${ }^{3,4}$ Chris Blair*, ${ }^{2}$ Geoffrey Parker, ${ }^{2}$ David Brunacci, ${ }^{4,2} \mathrm{Tim}$ Ang, ${ }^{5}$ John Worthington, ${ }^{6}$ Prakash Muthusami, ${ }^{1}$ Timo Krings. ${ }^{1}$ Department of Interventional Neuroradiology, Toronto Western Hospital, Toronto, Ontario, Canada; ${ }^{2}$ Department of Interventional Neuroradiology, Royal Prince Alfred Hospital, Sydney, NSW, Australia; ${ }^{3}$ Department of Neurology, Liverpool Hospital, Liverpool, NSW, Australia; ${ }^{4}$ Department of Neurology, Royal Prince Alfred Hospital, Camperdown, NSW, Australia; ${ }^{5}$ Department of Neurology, Royal Prince Alfred Hospital, Sydney, NSW, Australia; ${ }^{6}$ Department of Interventional Radiology, The Hospital for Sick Children, Toronto, Ontario, Canada
\end{abstract}

\subsection{6/jnnp-2019-anzan.12}

Introduction In adults, there is strong evidence demonstrating the superiority of mechanical thrombectomy (MT) plus intravenous thrombolysis over thrombolysis alone for the treatment of acute ischemic stroke due to large vessel occlusion (LVO). The role of MT in the paediatric stroke population is less clear. Here we present an updated systematic review addressing the use of MT in paediatric patients, including three cases from our centre in Sydney, Australia. We have also completed an individual participant data (IPD) meta-analysis of clinical and angiographic outcomes based on these results.

Method Our systematic review and IPD meta-analysis was performed according to PRISMA-IPD (Preferred Reporting Items for Systematic Reviews and Meta-Analyses: Individual Participant Data) guidelines. Primary outcomes measures were change in NIHSS (National Institute of Health Stroke Scale) score following MT, and mRS (modified Rankin Scale) score at final reported follow-up. The secondary outcome measure was final angiographic result using the mTICI (modified Treatment in Cerebral Ischemia) scale.

Results MT resulted in good long-term neurological outcomes (mRS 0-2) in 60/67 cases (89.6\%;follow-up timing $\mu=4.1$ months: 95\%CI 2.9-5.3), good short-term neurological outcomes (reduction in NIHSS by 8 or more points or post-MT NIHSS of $0-1$ ) in $37 / 52$ cases $(71.2 \%)$, and successful recanalization (mTICI $2 \mathrm{~b} / 3$ ) in $57 / 67$ cases $(85.1 \%$ ).

Conclusions

In paediatric patients, MT is an effective treatment for ischaemic stroke due to LVO. In the absence of a dedicated prospective registry and with randomized control trails unfeasible, this report represents the best available evidence for the use of MT in the paediatric setting.

\section{THE IMPACT OF AGGRESSIVE BLOOD PRESSURE MANAGEMENT IN THE POST-THROMBOLYSIS SETTING}

${ }^{1}$ Bethan Harper*, ${ }^{2}$ Harry McNaughton, ${ }^{3}$ Anna Ranta. ${ }^{1}$ Neurology, Capital and Coast District Health Board and University of Otago, Wellington, New Zealand; ${ }^{2}$ Medical Research Institute of New Zealand, Wellington, New Zealand; ${ }^{3}$ Capital and Coast District Health Board and University of Otago, Wellington, Wellington, New Zealand

\subsection{6/jnnp-2019-anzan.13}

Introduction High blood pressure (BP) post-thrombolysis has been associated with an increased rate of bleeding and poorer outcome. We noted frequent BPs of $>180 \mathrm{mmHg}$ with a target of keeping $\mathrm{BP}<180$. We tested whether a more aggressive target of SBP $<160 \mathrm{mmHg}$ would result in fewer BP protocol violations.

Methods Patients were prospectively captured comparing patients thrombolysed during the 12 months before and 12 months following the introduction of a new more aggressive $\mathrm{BP}$ protocol, allowing for a 6 month transition period. Results were adjusted for baseline function and stroke severity using regression analysis.

Results Pre-protocol change 68 and post- 100 patients were thrombolysed. Baseline characteristics were similar between groups. There was a trend for a lower rate of SBPs $>180$ $\mathrm{mmHg}$ (adjusted OR 0.49; 95\% CI 0.31-1.1; $\mathrm{p}=0.097$ ) and a significantly higher rate of SBPs $<120 \mathrm{mmHg}$ (adjusted OR 3.06 ; 95\% CI $1.52-6.17 ; \mathrm{p}=0.002$ ) in the aggressive BP protocol group; although events of extreme SBPs (>200 and $<100 \mathrm{mmHg}$ ) were similar between groups. Favourable outcomes (mRS $=0-2)$ at 3 months were similar between groups (adjusted OR 1.27; 95\% CI $0.58-2.8 ; \mathrm{p}=0.56$ ) as was the rate of symptomatic haemorrhages (adjusted OR 1.26;95\% CI 0.28-5.7; $\mathrm{p}=0.76$ ). Model fit was improved by adding study group to the model.

Conclusions More aggressive post-thrombolysis BP management lowered the overall BP, but did not result in improved patient outcomes. Potential explanations include a small sample size, reduced cerebral perfusion off-setting reduced bleeding risk, or high BP being merely an epiphenomenon of worse outcome rather than causative.

\section{AVXS-101 GENE-REPLACEMENT THERAPY (GRT) IN PRESYMPTOMATIC SPINAL MUSCULAR ATROPHY (SMA): STUDY UPDATE}

${ }^{1}$ Michelle Farrar* ${ }^{*}$ Kathryn J Swoboda, ${ }^{3}$ Meredith Schultz, ${ }^{4}$ Hugh McMillan, ${ }^{5}$ Julie Parsons, ${ }^{6}$ lan E Alexander, ${ }^{3}$ Elaine Kernbauer, ${ }^{3}$ Marcia Farrow, ${ }^{3}$ Francis G Ogrinc, ${ }^{3}$ Douglas E Feltner, ${ }^{3}$ Bryan E McGill, ${ }^{3}$ Sidney A Spector, ${ }^{3}$ James L'Italien, ${ }^{3}$ Douglas M Sproule, ${ }^{7}$ Kevin A Strauss. ${ }^{1}$ Department of Neurology, Sydney Children's Hospital, Randwick, NSW, Australia; ${ }^{2}$ Department of Neurology, Massachusetts General Hospital, Boston, MA, USA; ${ }^{3}$ AveXis, Inc., Bannockburn, IL, USA; ${ }^{4}$ Department of Pediatrics, Canada Children's Hospital of Eastern Ontario, Ontario, Ottawa, Canada; ${ }^{5}$ Department of Neurology, Children's Hospital Colorado, Aurora, CO, USA; ${ }^{6}$ Gene Therapy Research Unit, Children's Medical Research Institute and The Children's Hospital at Westmead, Sydney, NSW, Australia; ${ }^{7}$ Clinic for Special Children, Strasburg, PA, USA

10.1136/jnnp-2019-anzan. 14

Introduction SMA is a neurodegenerative disease caused by biallelic deletion/mutation of the survival motor neuron 1 